\title{
Bounded solutions of delay dynamic equations on time scales
}

Josef Diblík ${ }^{1,2^{*}}$ and Jiří Vítovec ${ }^{1}$

${ }^{\text {*Correspondence: }}$

diblik.j@fce.vutbr.cz;

diblik@feec.vutbr.cz

${ }^{1}$ Department of Mathematics,

Faculty of Electrical Engineering and Communications, Brno University of

Technology, Brno, Czech Republic

${ }^{2}$ Department of Mathematics and

Descriptive Geometry, Faculty of

Civil Engineering, Brno University of

Technology, Brno, Czech Republic

\begin{abstract}
In this paper we discuss the asymptotic behavior of solutions of the delay dynamic equation

$$
y^{\Delta}(t)=f(t, y(\tau(t)))
$$

where $f: \mathbb{T} \times \mathbb{R} \rightarrow \mathbb{R}, \tau: \mathbb{T} \rightarrow \mathbb{T}$ is a delay function and $\mathbb{T}$ is a time scale. We formulate the principle which gives the guarantee that the graph of at least one solution of the above mentioned equation stays in the prescribed domain. This principle uses the idea of the retraction method and is a suitable tool for investigating the asymptotic behavior of solutions of dynamic equations. This is illustrated by an example.
\end{abstract}

\section{Introduction}

\subsection{Time scale calculus}

We assume that the reader is familiar with the notion of time scales. Thus, note just that $\mathbb{T},[a, b]_{\mathbb{T}}:=[a, b] \cap \mathbb{T}$ (resp. $(a, b)_{\mathbb{T}}:=(a, b) \cap \mathbb{T}$ or similarly, we define any combination of right and left open or closed interval), $[a, \infty)_{\mathbb{T}}:=[a, \infty) \cap \mathbb{T}, \sigma, \rho, \mu$ and $f^{\Delta}$ stand for the time scale, a finite time scale interval, an infinite time scale interval, a forward jump operator, a backward jump operator, graininess and a $\Delta$-derivative of $f$. Further, the symbols $C(\mathbb{T}), C_{\mathrm{rd}}(\mathbb{T})$ and $C_{\mathrm{rd}}^{1}(\mathbb{T})$ stand for the class of continuous, rd-continuous and rd-continuous $\Delta$-derivative functions. See [1], which is the initiating paper of the time scale theory, the thesis [2] and [3] containing a lot of information on time scale calculus.

Now, we remind further aspects of time scale calculus, which will be needed later; see, e.g., [3].

Definition 1 Let $\mathbb{T}$ be a time scale. A function $f: \mathbb{T} \times \mathbb{R} \rightarrow \mathbb{R}$ is called

(i) $r d$-continuous, if $g$ defined by $g(t):=f(t, y(t))$ is rd-continuous for any rd-continuous function $y: \mathbb{T} \rightarrow \mathbb{R}$;

(ii) bounded on a set $S \subset \mathbb{T} \times \mathbb{R}$, if there exists a constant $M>0$ such that

$$
|f(t, y)| \leq M \quad \text { for all }(t, y) \in S
$$

(iii) Lipschitz continuous on a set $S \subset \mathbb{T} \times \mathbb{R}$, if there exists a constant $L>0$ such that

$$
\left|f\left(t, y_{1}\right)-f\left(t, y_{2}\right)\right| \leq L\left|y_{1}-y_{2}\right| \quad \text { for all }\left(t, y_{1}\right),\left(t, y_{2}\right) \in S .
$$

\section{Springer}

(c) 2012 Diblík and Vítovec; licensee Springer. This is an Open Access article distributed under the terms of the Creative Commons Attribution License (http://creativecommons.org/licenses/by/2.0), which permits unrestricted use, distribution, and reproduction in any medium, provided the original work is properly cited. 


\subsection{Delay dynamic equations on time scales}

Let $\tau: \mathbb{T} \rightarrow \mathbb{T}$ be an increasing rd-continuous function satisfying $\tau(t) \leq t$ for all $t \in \mathbb{T}$. Let the function $f: \mathbb{T} \times \mathbb{R} \rightarrow \mathbb{R}$ be rd-continuous. We consider the delay dynamic equation

$$
y^{\Delta}(t)=f(t, y(\tau(t)))
$$

on time scales $\mathbb{T}$. (Note that $y(\tau(t)) \in C_{\mathrm{rd}}(\mathbb{T})$ in view of Definition 1.)

For a given $t_{0} \in \mathbb{T}$, a function $y:\left[\tau\left(t_{0}\right), \infty\right)_{\mathbb{T}} \rightarrow \mathbb{R}$ is said to be a solution of (1) on $\left[\tau\left(t_{0}\right), \infty\right)_{\mathbb{T}}$ provided $y \in C_{\mathrm{rd}}\left(\left[\tau\left(t_{0}\right), \infty\right)_{\mathbb{T}}\right), y \in C_{\mathrm{rd}}^{1}\left(\left[t_{0}, \infty\right)_{\mathbb{T}}\right)$ and $y$ satisfies (1) for all $t \in\left[t_{0}, \infty\right)_{\mathbb{T}}$. If, moreover, an initial function $\varphi \in C\left(\left[\tau\left(t_{0}\right), t_{0}\right]_{\mathbb{T}}\right)$ be given and

$$
y(t)=\varphi(t), \quad t \in\left[\tau\left(t_{0}\right), t_{0}\right]_{\mathbb{T}},
$$

then we say that $y$ is a solution of the initial value problem (IVP) (1) and (2).

\subsection{Existence and uniqueness of solutions of delay dynamic equations}

For the next study, it is important to verify the existence and uniqueness of solutions of IVP (1) and (2). However, the following theorem (in a more general form) can be found in $[4$, Theorem 2.1].

Theorem 1 (Picard-Lindelöf theorem) Let $t_{1} \in \mathbb{T}, t_{1}>t_{0}, m>0$. Let

$$
Y_{m}:=\left\{y \in \mathbb{R}:|y-\varphi(t)| \leq m \text { for all } t \in\left[\tau\left(t_{0}\right), t_{0}\right]_{\mathbb{T}}\right\},
$$

where the properties of $\varphi$ are described in previous Section 1.2. Assume that $f \in C_{\mathrm{rd}}\left(\left[t_{0}\right.\right.$, $\left.\left.t_{1}\right]_{\mathbb{T}} \times Y_{m}\right)$ is bounded on $\left[t_{0}, t_{1}\right]_{\mathbb{T}} \times Y_{m}$, with bound $M>0$, and Lipschitz continuous on $\left[t_{0}, t_{1}\right]_{\mathbb{T}} \times Y_{m}$. Then the initial value problem (1) and (2) has a unique solution $y$ on the interval $\left[\tau\left(t_{0}\right), \sigma(\xi)\right]_{\mathbb{T}} \subset\left[\tau\left(t_{0}\right), t_{1}\right]_{\mathbb{T}}$, where

$$
\xi:=\max \left[t_{0}, t_{0}+\delta\right]_{\mathbb{T}}
$$

and

$$
\delta:=\min \left\{t_{1}-t_{0}, m / M\right\}
$$

Carefully tracing the proof of Theorem 1 in [4], it easy to verify that if Theorem 1 holds, then the solution of the IVP (1) and (2) depends continuously on the initial data.

\section{Problem under consideration}

Let $b, c: \mathbb{T} \rightarrow \mathbb{R}$ be rd-continuous functions such that $b(t)<c(t)$ for all $t \in\left[\tau\left(t_{0}\right), \infty\right]_{\mathbb{T}}$ and

$$
b(t)<\varphi(t)<c(t) \quad \text { for all } t \in\left[\tau\left(t_{0}\right), t_{0}\right]_{\mathbb{T}} .
$$

We define a set $\Omega \subset \mathbb{T} \times \mathbb{R}$ as

$$
\Omega:=\left\{(t, y): t \in\left[\tau\left(t_{0}\right), \infty\right]_{\mathbb{T}}, b(t)<y<c(t)\right\} .
$$


Then the closure $\bar{\Omega}$ equals

$$
\bar{\Omega}:=\left\{(t, y): t \in\left[\tau\left(t_{0}\right), \infty\right]_{\mathbb{T}}, b(t) \leq y \leq c(t)\right\}
$$

and the boundary $\partial \Omega=\partial \Omega_{B} \cup \partial \Omega_{C}$, where

$$
\partial \Omega_{B}:=\left\{(t, y): t \in\left[\tau\left(t_{0}\right), \infty\right]_{\mathbb{T}}, y=b(t)\right\}
$$

and

$$
\partial \Omega_{C}:=\left\{(t, y): t \in\left[\tau\left(t_{0}\right), \infty\right]_{\mathbb{T}}, y=c(t)\right\}
$$

Consider the delay dynamic equation (1) and the initial value problem (2). Let

$$
b_{*}:=\min \left\{b(t): t \in\left[\tau\left(t_{0}\right), t_{0}\right]_{\mathbb{T}}\right\}
$$

and

$$
c^{*}:=\max \left\{c(t): t \in\left[\tau\left(t_{0}\right), t_{0}\right]_{\mathbb{T}}\right\} .
$$

Let $t_{1} \in \mathbb{T}, t_{1}>t_{0}$. Throughout, we will assume that a function $f$ is bounded and Lipschitz continuous on a domain $S=S(t, y) \subset \mathbb{T} \times \mathbb{R}$ and

$$
\left\{\left[\tau\left(t_{0}\right), t_{1}\right]_{\mathbb{T}} \times\left[b_{*}, c^{*}\right]\right\} \cap \bar{\Omega} \subset S .
$$

This condition says that by Theorem 1 every initial value problem (1) and (2) with $\varphi$ satisfying (3) has exactly one solution on the interval $\left[\tau\left(t_{0}\right), \sigma(\xi)\right]_{\mathbb{T}}, \sigma(\xi)>t_{0}$. It is also easy to show that this solution depends continuously on the initial function $\varphi$.

Our aim is to establish sufficient conditions for the right-hand side of equation (1) in order to guarantee the existence of at least one solution $y(t)$ of $(1)$ defined on $\left[\tau\left(t_{0}\right), \infty\right]_{\mathbb{T}}$ such that $(t, y(t)) \in \Omega$ for each $t \in\left[\tau\left(t_{0}\right), \infty\right]_{\mathbb{T}}$. The main result generalizes some previous results of the first author (and his co-authors) concerning the asymptotic behavior of solutions of discrete equations; see, e.g., [5-16]. In papers [5, 7], in our best knowledge, the retract principle is extended to discrete equations (see [9-11] as well). In $[6,8,14,16]$ delayed discrete equations are considered by the retract technique, and in [12] the retract principle is given for discrete time scales. Papers $[13,15]$ are devoted to the extension of the retract principle to dynamic equations. In the present paper, we give an attempt to enlarge the retract principle to delayed dynamic equations.

For further consideration, it is convenient to establish the following concept.

Definition 2 A point $M=(t, b(t)) \in \partial \Omega_{B}, t \geq t_{0}$, is called the point of strict egress for the set $\Omega$ with respect to equation (1) if

$$
f(t, \psi(\tau(t)))<b^{\Delta}(t)
$$

where $\psi:[\tau(t), t]_{\mathbb{T}} \rightarrow \mathbb{R}$ is an arbitrary $r d$-continuous function such that $b(s)<\psi(s)<c(s)$ for every $s \in[\tau(t), t)_{\mathbb{T}}$ and $\psi(t)=b(t)$. 
A point $M=(t, c(t)) \in \partial \Omega_{C}, t \geq t_{0}$, is called the point of strict egress for the set $\Omega$ with respect to equation (1) if

$$
f(t, \psi(\tau(t)))>c^{\Delta}(t)
$$

where $\psi:[\tau(t), t]_{\mathbb{T}} \rightarrow \mathbb{R}$ is an arbitrary $r d$-continuous function such that $b(s)<\psi(s)<c(s)$ for every $s \in[\tau(t), t)_{\mathbb{T}}$ and $\psi(t)=c(t)$.

Remark 1 The geometrical meaning of the point of strict egress is evident. If a point $\left(t_{*}, b\left(t_{*}\right)\right) \in \partial \Omega_{B}$ is a point of strict egress for the set $\Omega$ with respect to (1), and $y(t)$ is a (unique) solution of (1) satisfying $y\left(t_{*}\right)=b\left(t_{*}\right)$, then due to (4),

$$
\left(y\left(t_{*}\right)-b\left(t_{*}\right)\right)^{\Delta}=f\left(t_{*}, \psi\left(\tau\left(t_{*}\right)\right)\right)-b^{\Delta}\left(t_{*}\right)<0 .
$$

From the definition of a $\Delta$-derivative, we get $y(t)-b(t)<0($ or $(t, y(\tau(t))) \notin \bar{\Omega})$ for $t \in$ $\left(t_{*}, t_{*}+\delta\right)_{\mathbb{T}}$ with a small positive $\delta$ if $t_{*}$ is a right-dense point and for $t=\sigma\left(t_{*}\right)$ if $t_{*}$ is rightscattered.

By analogy, if $\left(t^{*}, c\left(t^{*}\right)\right) \in \partial \Omega_{C}$ is a point of strict egress for the set $\Omega$ with respect to (1), and $y(t)$ is a (unique) solution of (1) satisfying $y\left(t^{*}\right)=c\left(t^{*}\right)$, then due to (5),

$$
\left(y\left(t^{*}\right)-c\left(t^{*}\right)\right)^{\Delta}=f\left(t^{*}, \psi\left(\tau\left(t^{*}\right)\right)\right)-c^{\Delta}\left(t^{*}\right)>0 .
$$

From the definition of a $\Delta$-derivative, we get $y(t)-c(t)>0($ or $(t, y(t)) \notin \bar{\Omega})$ for $t \in\left(t^{*}, t^{*}+\right.$ $\delta)_{\mathbb{T}}$ with a small positive $\delta$ if $t^{*}$ is a right-dense point and for $t=\sigma\left(t^{*}\right)$ if $t^{*}$ is right-scattered.

Definition 3 ([17]) If $A \subset B$ are subsets of a topological space and $\pi: B \rightarrow A$ is a continuous mapping from $B$ onto $A$ such that $\pi(p)=p$ for every $p \in A$, then $\pi$ is said to be a retraction of $B$ onto $A$. When a retraction of $B$ onto $A$ exists, $A$ is called a retract of $B$.

\section{Existence theorem}

The proof of the following theorem is based on the retract method, which is well known for ordinary differential equations and goes back to Ważewski [18]. Below we will assume that the function $f$, except for the indicated conditions, satisfies all the assumptions given in Section 2.

Theorem 2 Let $f: \mathbb{T} \times \mathbb{R} \rightarrow \mathbb{R}$. Let $b, c: \mathbb{T} \rightarrow \mathbb{R}$ be delta differentiable functions such that $b(t)<c(t)$ for each $t \in\left[\tau\left(t_{0}\right), \infty\right)_{\mathbb{T}}$. If, moreover, every point $M \in \partial \Omega_{B} \cup \partial \Omega_{C}$ is the point of strict egress for the set $\Omega$ with respect to equation (1), then there exists an $r d$-continuous initial function $\varphi^{*}:\left[\tau\left(t_{0}\right), t_{0}\right]_{\mathbb{T}} \rightarrow \mathbb{R}$ satisfying

$$
b(t)<\varphi^{*}(t)<c(t) \quad \text { for all } t \in\left[\tau\left(t_{0}\right), t_{0}\right]_{\mathbb{T}},
$$

such that the initial problem

$$
y(t)=\varphi^{*}(t), \quad t \in\left[\tau\left(t_{0}\right), t_{0}\right]_{\mathbb{T}}
$$


defines a solution $y$ of $(1)$ on the interval $\left[\tau\left(t_{0}\right), \infty\right)_{\mathbb{T}}$ satisfying

$$
b(t)<y(t)<c(t) \quad \text { for all } t \in\left[\tau\left(t_{0}\right), \infty\right)_{\mathbb{T}} .
$$

Proof The idea of the proof is simple. We suppose that the statement of the theorem is not valid. Then it is possible to prove that there exists a retraction of a segment $B:=[\alpha, \beta]$ with $\alpha<\beta$ onto a two-point set $A:=\{\alpha, \beta\}$. But it is well known that the boundary of a nonempty (closed) interval cannot be its retract (see [19]). So, in our case, such a retractive mapping cannot exist because it is incompatible with continuity.

Without any special comment, throughout the proof, we use the property that the initial value problem in question has a unique solution and the property of continuous dependence of solutions on their initial data.

Suppose now that $\varphi^{*}$ satisfying the inequality

$$
b(t)<\varphi^{*}(t)<c(t) \quad \text { for all } t \in\left[\tau\left(t_{0}\right), t_{0}\right]_{\mathbb{T}}
$$

and generating the solution $y=y(t)$ which satisfies (7) for any $t \in\left[\tau\left(t_{0}\right), \infty\right)_{\mathbb{T}}$ does not exist. This means that for any $r d$-continuous initial function $\varphi_{0}$ satisfying the inequality

$$
b(t)<\varphi_{0}(t)<c(t) \quad \text { for all } t \in\left[\tau\left(t_{0}\right), t_{0}\right]_{\mathbb{T}},
$$

there exists a $t^{0} \in \mathbb{T}, t^{0}>t_{0}$ such that for a corresponding solution $y=y^{0}(t)$ of the initial problem

$$
y^{0}(t)=\varphi_{0}(t), \quad t \in\left[\tau\left(t_{0}\right), t_{0}\right]_{\mathbb{T}}
$$

we have

$$
\left(t^{0}, y^{0}\left(t^{0}\right)\right) \notin \Omega
$$

and,

$$
\left(t, y^{0}(t)\right) \in \Omega \quad \text { for all } t \in\left[t_{0}, t^{0}\right)_{\mathbb{T}} .
$$

Let us define auxiliary mappings $P_{1}, P_{2}$ and $P_{3}$.

First, define the mapping $P_{1}: B_{1} \rightarrow \mathbb{T} \times \mathbb{R}$, where

$$
B_{1}=\left\{\left(t_{0}, \varphi_{0}\left(t_{0}\right)\right) \in \mathbb{T} \times \mathbb{R}: b\left(t_{0}\right) \leq \varphi_{0}\left(t_{0}\right) \leq c\left(t_{0}\right)\right\},
$$

such that

(i) for $\varphi_{0}(t), b(t)<\varphi_{0}(t)<c(t), t \in\left[\tau\left(t_{0}\right), t_{0}\right]_{\mathbb{T}}$, we define

$$
P_{1}:\left(t_{0}, \varphi_{0}\left(t_{0}\right)\right) \rightarrow\left(t^{0}, y^{0}\left(t^{0}\right)\right)
$$

(ii) for $\varphi_{0}(t)$ satisfying $b(t)<\varphi_{0}(t)<c(t), t \in\left[\tau\left(t_{0}\right), t_{0}\right)_{\mathbb{T}}$ and $\varphi_{0}\left(t_{0}\right)=b\left(t_{0}\right)$, we put $t^{0}=t_{0}$ and define

$$
P_{1}:\left(t_{0}, \varphi_{0}\left(t_{0}\right)\right) \rightarrow\left(t^{0}, y^{0}\left(t^{0}\right)\right)=\left(t_{0}, b\left(t_{0}\right)\right)
$$


(iii) for $\varphi_{0}(t), b(t)<\varphi_{0}(t)<c(t), t \in\left[\tau\left(t_{0}\right), t_{0}\right)_{\mathbb{T}}$ and $\varphi_{0}\left(t_{0}\right)=c\left(t_{0}\right)$, we put $t^{0}=t_{0}$ and define

$$
P_{1}:\left(t_{0}, \varphi_{0}\left(t_{0}\right)\right) \rightarrow\left(t^{0}, y^{0}\left(t^{0}\right)\right)=\left(t_{0}, c\left(t_{0}\right)\right)
$$

Second, we define the mapping $P_{2}: B_{2} \rightarrow \mathbb{T} \times \mathbb{R}$, where

$$
B_{2}=P\left(B_{1}\right)=\left\{\left(t^{0}, y^{0}\left(t_{0}\right)\right) \in \mathbb{T} \times \mathbb{R}: y^{0}\left(t_{0}\right) \leq b\left(t^{0}\right) \text { or } y^{0}\left(t_{0}\right) \geq c\left(t^{0}\right)\right\},
$$

as

$$
P_{2}:\left(t^{0}, y^{0}\left(t^{0}\right)\right) \rightarrow \begin{cases}\left(t^{0}, c\left(t^{0}\right)\right) & \text { if } y^{0}\left(t^{0}\right) \geq c\left(t^{0}\right) \\ \left(t^{0}, b\left(t^{0}\right)\right) & \text { if } y^{0}\left(t^{0}\right) \leq b\left(t^{0}\right)\end{cases}
$$

Third, we define the mapping $P_{3}: B_{3} \rightarrow \mathbb{T} \times \mathbb{R}$, where

$$
B_{3}=P\left(B_{2}\right)=\left\{\left(t^{0}, \tilde{y}\right) \in \mathbb{T} \times \mathbb{R}: \tilde{y}=b\left(t^{0}\right) \text { or } \tilde{y}=c\left(t^{0}\right)\right\},
$$

as

$$
P_{3}:\left(t^{0}, \tilde{y}\right) \rightarrow \begin{cases}\left(t_{0}, c\left(t_{0}\right)\right) & \text { if } \tilde{y}=c\left(t^{0}\right), \\ \left(t_{0}, b\left(t_{0}\right)\right) & \text { if } \tilde{y}=b\left(t^{0}\right) .\end{cases}
$$

We will show that the composite mapping

$$
P:=P_{3} \circ P_{2} \circ P_{1}, \quad P: B_{1} \rightarrow A_{1},
$$

where

$$
A_{1}=\left\{\left(t_{0}, b\left(t_{0}\right)\right),\left(t_{0}, c\left(t_{0}\right)\right)\right\},
$$

is continuous with respect to the second coordinate $\varphi_{0}\left(t_{0}\right)$ of the point $\left(t_{0}, \varphi_{0}\left(t_{0}\right)\right) \in B_{1}$. The definition of the mapping $P$ implies that only two resulting points are possible, namely either $P\left(B_{1}\right)=\left(t_{0}, c\left(t_{0}\right)\right)$ or $P\left(B_{1}\right)=\left(t_{0}, b\left(t_{0}\right)\right)$.

(I) We consider the first possibility, i.e., $P\left(B_{1}\right)=\left(t_{0}, c\left(t_{0}\right)\right)$. Let $b(t)<\varphi_{0}(t)<c(t)$ for all $t \in\left[\tau\left(t_{0}\right), t_{0}\right)_{\mathbb{T}}$. Then

$$
P_{1}\left(t_{0}, \varphi_{0}\left(t_{0}\right)\right)=\left(t^{0}, y^{0}\left(t^{0}\right)\right),\left(t^{0}, y^{0}\left(t^{0}\right)\right) \notin \Omega \quad \text { and } \quad y^{0}\left(t^{0}\right) \geq c\left(t^{0}\right) .
$$

Let $y^{0}\left(t^{0}\right)>c\left(t^{0}\right)$. Then $\rho\left(t^{0}\right)<t^{0}$ and the continuity of the mapping $P_{1}$ is obvious. Indeed, if

$$
\varphi^{0, \varepsilon}(t), \quad t \in\left[\tau\left(t_{0}\right), t_{0}\right], \quad b(t)<\varphi^{0, \varepsilon}(t)<c(t), \quad t \in\left[\tau\left(t_{0}\right), t_{0}\right)
$$

is the initial problem defining the solution $y^{\varepsilon}(t), \varepsilon$ is a sufficiently small number and

$$
\left|\varphi^{0, \varepsilon}(t)-\varphi_{0}(t)\right|<\varepsilon, \quad t \in\left[\tau\left(t_{0}\right), t_{0}\right],
$$


then due to the property of continuous dependence of solutions on their initial data, $t^{0, \varepsilon}=$ $t^{0}$ and

$$
P_{1}\left(t_{0}, \varphi^{0, \varepsilon}\left(t_{0}\right)\right)=\left(t^{0, \varepsilon}, y^{\varepsilon}\left(t^{0, \varepsilon}\right)\right)=\left(t^{0}, y^{\varepsilon}\left(t^{0}\right)\right) \quad \text { with } \quad y^{\varepsilon}\left(t^{0}\right)>c\left(t^{0}\right) .
$$

Consequently, $P\left(t_{0}, \varphi^{0, \varepsilon}\left(t_{0}\right)\right)=\left(t_{0}, c\left(t_{0}\right)\right)$.

Let $y^{0}\left(t^{0}\right)=c\left(t^{0}\right)$. By the assumption of the theorem, every boundary point of $\partial \Omega$ is the point of strict egress for the set $\Omega$ with respect to equation (1). Then for the solution $y^{\varepsilon}(t)$ defined by (9), we have

$$
P_{1}\left(t_{0}, \varphi^{0, \varepsilon}\left(t_{0}\right)\right)=\left(t^{0, \varepsilon}, y^{\varepsilon}\left(t^{0, \varepsilon}\right)\right)
$$

either with $y^{\varepsilon}\left(t^{0, \varepsilon}\right)>c\left(t^{0, \varepsilon}\right)$ or with $t^{0, \varepsilon}=t^{0}, y^{\varepsilon}\left(t^{0, \varepsilon}\right)=c\left(t_{0}\right)$. (We do not describe all the possibilities for the occurrence of the first or of the second alternative.) In both alternatives we get $P\left(t_{0}, \varphi^{0, \varepsilon}\left(t_{0}\right)\right)=\left(t_{0}, c\left(t_{0}\right)\right)$ again. Hence, the mapping $P$ is continuous in the considered case.

(II) We proceed analogously with the case $P\left(B_{1}\right)=\left(t_{0}, b\left(t_{0}\right)\right)$.

The continuity of the mapping $P$ was proved for initial functions $\varphi_{0}$ satisfying $b(t)<$ $\varphi_{0}(t)<c(t)$ for all $t \in\left[\tau\left(t_{0}\right), t_{0}\right)_{\mathbb{T}}$ and $b\left(t_{0}\right)<\varphi_{0}\left(t_{0}\right)<c\left(t_{0}\right)$. The desired retraction $P_{r}$ can be defined as a mapping of the second coordinates realized by $P$. Then the mapping

$$
\left[b\left(t_{0}\right), c\left(t_{0}\right)\right] \stackrel{P_{r}}{\longrightarrow}\left\{b\left(t_{0}\right), c\left(t_{0}\right)\right\}
$$

is continuous and

$$
\left\{b\left(t_{0}\right)\right\} \stackrel{P_{r}}{\longrightarrow}\left\{b\left(t_{0}\right)\right\}, \quad\left\{c\left(t_{0}\right)\right\} \stackrel{P_{r}}{\longrightarrow}\left\{c\left(t_{0}\right)\right\},
$$

i.e., the points $\left\{b\left(t_{0}\right)\right\},\left\{c\left(t_{0}\right)\right\}$ are stationary.

In this situation we proved that there exists a retraction $P_{r}$ of the set $B:=\left[b\left(t_{0}\right), c\left(t_{0}\right)\right]$ onto the two-point set $A:=\left\{b\left(t_{0}\right), c\left(t_{0}\right)\right\}$ (see Definition 3). In regard to the above mentioned fact, this is impossible. Our supposition is false, and there exists the initial problem (6) such that the corresponding solution $y=y^{*}(t)$ satisfies the inequalities (7) for every $t \in\left[\tau\left(t_{0}\right), \infty\right]_{\mathbb{T}}$. The theorem is proved.

\section{Example}

Let us consider the delay dynamic equation of the type (1)

$$
y^{\Delta}=f(t, y(\tau(t))):=\frac{1}{t^{3}} \cdot y(\tau(t))+\frac{\sin (y(\tau(t)))}{1+t^{3}}+\frac{\cos (y(\tau(t)))}{1+t^{3}}
$$

defined for each $t \in[a, \infty)_{\mathbb{T}}$ with $a \in \mathbb{T}, \tau(a)>1$ and $\mu(t)=O(t)$ (which means that there exists $d>0$ such that $\mu(t) \leq d t$ for each $t \in \mathbb{T})$. Note that we have no further requirements for the function $\tau$ (except for those mentioned in Section 1.2). Moreover, let $t_{0} \in \mathbb{T}, t_{0}>a$ be sufficiently large. With the aid of Theorem 1 , we will show that there exists an initial function

$$
\varphi^{*}(t) \in\left(-t^{-1}, t^{-1}\right), \quad t \in\left[\tau\left(t_{0}\right), t_{0}\right]_{\mathbb{T}},
$$


which defines a solution $y(t)$ for all $t \in\left[\tau\left(t_{0}\right), \infty\right){ }_{\mathbb{T}}$ of the dynamic equation (10) satisfying

$$
|y(t)|<t^{-1}
$$

We define $\Delta$-differentiable functions $b, c:\left[\tau\left(t_{0}\right), \infty\right)_{\mathbb{T}} \rightarrow \mathbb{R}$ satisfying $b(t)<c(t)$ for each $t \in \mathbb{T}$ as

$$
b(t):=-t^{-1}, \quad c(t):=t^{-1} .
$$

We will verify that every point $M \in \partial \Omega=\partial \Omega_{B} \cup \partial \Omega_{C}$, where

$$
\begin{aligned}
& \partial \Omega_{B}:=\left\{(t, y): t \in\left[\tau\left(t_{0}\right), \infty\right)_{\mathbb{T}}, y=-t^{-1}\right\}, \\
& \partial \Omega_{C}:=\left\{(t, y): t \in\left[\tau\left(t_{0}\right), \infty\right)_{\mathbb{T}}, y=t^{-1}\right\},
\end{aligned}
$$

is a point of strict egress for the set

$$
\Omega:=\left\{(t, y): t \in\left[\tau\left(t_{0}\right), \infty\right)_{\mathbb{T}},-t^{-1}<y<t^{-1}\right\}
$$

with respect to the dynamic equation (10).

For an arbitrary function $\psi:[\tau(t), t]_{\mathbb{T}} \rightarrow \mathbb{R}, t \in\left[t_{0}, \infty\right)_{\mathbb{T}}$ such that $b(s)<\psi(s)<c(s)$, $s \in[\tau(t), t)_{\mathbb{T}}$ and $\psi(t)=b(t)$, we need (see (4))

$$
\frac{1}{t^{3}} \cdot \psi(\tau(t))+\frac{\sin (\psi(\tau(t)))}{1+t^{3}}+\frac{\cos (\psi(\tau(t)))}{1+t^{3}}<\left(-\frac{1}{t}\right)^{\Delta}
$$

and analogously, for an arbitrary function $\psi:[\tau(t), t]_{\mathbb{T}} \rightarrow \mathbb{R}, t \in\left[t_{0}, \infty\right)_{\mathbb{T}}$ such that $b(s)<$ $\psi(s)<c(s), s \in[\tau(t), t)_{\mathbb{T}}$ and $\psi(t)=c(t)$, we need (see (5))

$$
\frac{1}{t^{3}} \cdot \psi(\tau(t))+\frac{\sin (\psi(\tau(t)))}{1+t^{3}}+\frac{\cos (\psi(\tau(t)))}{1+t^{3}}>\left(\frac{1}{t}\right)^{\Delta} .
$$

Inequalities (13) and (14) will be valid if

$$
\frac{1}{t^{3}}+\frac{2}{1+t^{3}}<\frac{1}{t^{2}(1+d)}
$$

Indeed, (we underline that for $t_{0}$ sufficiently large the last inequality holds) we have

$$
\begin{aligned}
\left|\frac{1}{t^{3}} \cdot \psi(\tau(t))+\frac{\sin (\psi(\tau(t)))}{1+t^{3}}+\frac{\cos (\psi(\tau(t)))}{1+t^{3}}\right| & <\frac{1}{t^{3}}+\frac{2}{1+t^{3}}<\frac{1}{t^{2}(1+d)} \\
& <\left|\left(\frac{1}{t}\right)^{\Delta}\right|=\frac{1}{t(t+\mu(t))} .
\end{aligned}
$$

Hence, in view of Definition 2, every point $M \in \partial \Omega$ is a point of strict egress for the set $\Omega$. Therefore, all the assumptions of Theorem 1 hold and there exists an initial value function $\varphi^{*}$ with the property (11) such that the initial problem $y(t)=\varphi^{*}(t)$ defines a solution $y$ on the interval $\mathbb{T}=\left[\tau\left(t_{0}\right), \infty\right)_{\mathbb{T}}$ of $(10)$ satisfying the inequality (12) for every $t \in\left[\tau\left(t_{0}\right), \infty\right)_{\mathbb{T}}$. Note that this solution (due to (12)) tends to zero as $t \rightarrow \infty$. 
Remark 2 In our example of equation (1) with bounded and vanishing solution, the graininess $\mu(t)$ plays an important role. Roughly speaking, 'the bigger' the graininess will be, 'the harder' it will be to construct an example of equation (1) with a bounded solution. This follows from the formulas (4) and (5), where the function $f$ is between $\Delta$-derivatives of two functions, and these derivatives decrease to zero if $\mu(t)$ goes to infinity. However, in the cases (as is, e.g., the example above) the graininess satisfying $\mu(t)=O(t)$ is 'sufficiently big' and covers every well-known case of time scales (e.g., $\mathbb{T}=\mathbb{R}, \mathbb{T}=\mathbb{Z}, \mathbb{T}=h \mathbb{Z}, h>0$, and $\left.\mathbb{T}=q^{\mathbb{N}_{0}}, q>1\right)$.

\section{Competing interests}

The authors declare that they have no competing interests.

\section{Authors' contributions}

The authors have made the same contribution. All authors read and approved the final manuscript.

\section{Acknowledgements}

The research was supported by the Grant P201/10/1032 of the Czech Grant Agency (Prague), by 'Operational Programme Research and Development for Innovations', No. CZ.1.05/2.1.00/03.0097, as an activity of the regional Centre AdMaS, and by the Grant FEKT-S-11-2-921 of Faculty of Electrical Engineering and Communication, Brno University of Technology.

Received: 21 July 2012 Accepted: 9 October 2012 Published: 24 October 2012

\section{References}

1. Hilger, S: Analysis on measure chains - a unified approach to continuous and discrete calculus. Results Math. 18, 18-56 (1990)

2. Hilger, S: Ein Masskettenkalkül mit Anwendung auf Zentrumsmannigfaltigkeiten. PhD thesis, Universität Würzburg (1988)

3. Bohner, M, Peterson, A: Dynamic Equations on Time Scales. Birghäuser, Basel (2001)

4. Karpuz, B: Existence and uniqueness of solutions to systems of delay dynamic equations on time scales. Int. J. Math. Comput. 10(M11), 48-58 (2011) (Special volume)

5. Diblík, J: Retract principle for difference equations. In: Elaydi, S, Ladas, G, Popenda, J, Rakowski, J (eds.) Communications in Difference Equations: Proceedings of the Fourth International Conference on Difference Equations, Poznan, Poland, August 27-31, 1998, pp. 107-114. Gordon \& Breach, New York (2000)

6. Diblík, J, Hlavičková, I: Asymptotic behavior of solutions of delayed difference equations. Abstr. Appl. Anal. 2011, Article ID 671967 (2011). doi:10.1155/2011/671967

7. Diblík, J: Discrete retract principle for systems of discrete equations. Comput. Math. Appl. 42, 515-528 (2001)

8. Baštinec, J, Diblík, J, Zhang, B: Existence of bounded solutions of discrete delayed equations. In: Proceedings of the Sixth International Conference on Difference Equations, pp. 359-366. CRC Press, Boca Raton (2004)

9. Diblík, J: Anti-Lyapunov method for systems of discrete equations. Nonlinear Anal. 57, 1043-1057 (2004)

10. Diblík, J: Asymptotic behavior of solutions of discrete equations. Funct. Differ. Equ. 11, 37-48 (2004)

11. Diblík, J, Migda, M, Schmeidel, E: Bounded solutions of nonlinear discrete equations. Nonlinear Anal. 65, 845-853 (2006)

12. Diblík, J, Růžičková, M, Václavíková, B: A retract principle on discrete time scales. Opusc. Math. 26(3), 445-455 (2006)

13. Diblík, J, Růžičková, M, Šmarda, Z: Ważewski's method for systems of dynamic equations on time scales. Nonlinear Anal. 71, e1124-e1131 (2009)

14. Diblík, J, Hlavičková, I: Combination of Liapunov and retract methods in the investigation of the asymptotic behavior of solutions of systems of discrete equations. Dyn. Syst. Appl. 18(3-4), 507-537 (2009)

15. Diblík, J, Rưžičková, M, Václavíková, B: Bounded solutions of dynamic equations on time scales. Int. J. Differ. Equ. 3(1) 61-69 (2008)

16. Diblík, J, Hlavičková, I: Asymptotic upper and lower estimates of a class of positive solutions of a discrete linear equation with a single delay. Abstr. Appl. Anal. 2012, Article ID 764351 (2012). doi:10.1155/2012/764351

17. Lakshmikantham, V, Leela, S: Differential and Integral Inequalities - Ordinary Differential Equations, vol. I. Academic Press, New York (1969)

18. Ważewski, T: Sur un principe topologique de l'examen de l'allure asymptotique des intégrales des équations différentielles ordinaires. Ann. Soc. Pol. Math. 20, 279-313 (1947)

19. Borsuk, K: Theory of Retracts. PWN, Warsaw (1967) 